\title{
Überangepasst und realitätsentrückt Zur Paradoxie der Theorie der deliberativen Demokratie in der EU*
}

\section{Kurzfassung}

Von der Demokratietheorie wird erwartet, dass sie fußend auf einer möglichst exakten empirischen Problembeschreibung normativ überzeugende Alternativen zur Weiterentwicklung demokratischer Ordnungen anbietet. In diesem Beitrag wird die These vertreten, dass die in der Debatte um legitimes Regieren jenseits des Nationalstaats geradezu eine hegemoniale Stellung beanspruchende Theorie der „deliberativen“ Demokratie dies nicht zu leisten vermag, weil sie in diesem Verwendungszusammenhang unter der eigentümlichen Paradoxie leidet, sowohl überangepasst, als auch realitätsentrückt zu sein. Sie ist überangepasst, weil sie die partiell zu beobachtende Entkopplung der supranationalen Entscheidungsträger nicht mehr als demokratisches Übel, sondern als normativen Mehrwert interpretiert. Sie ist realitätsentrückt, weil sie andererseits nicht erkennen will, dass die Interaktionsmodi in europäischen Entscheidungsprozessen nur in Ausnahmefällen den Grundsätzen deliberativer Demokratie entsprechen. Das spricht mithin nicht gegen diese Theorie an sich - wohl aber gegen ihre Inanspruchnahme als europäische Demokratietheorie.

* Ich bedanke mich bei den beiden anonymen Gutachtern, die mir sehr geholfen haben, den Text noch einmal (selbst)kritisch zu überdenken. Manches habe ich daraufhin geändert, einiges jedoch trotz möglicher Mängel beibehalten - für die ich selbstverständlich die alleinige Verantwortung trage. 


\section{Inhalt}

1. Einleitung

2. Paradigmenwechsel in der Debatte um legitimes Europäisches

Regieren: Von der „komplexen“ zur „deliberativen“

Demokratietheorie

3. Deliberation statt Demokratie? Zur Anwendung der Theorie der deliberativen Demokratie im europäischen Kontext

4. Die EU als ,deliberative“" Demokratie?

5. Lackmustest: Zur ,,allgemeinen Zugänglichkeit“" deliberativer Prozesse in der EU

\section{Einleitung}

Stellvertretend für die politischen Wissenschaften in Deutschland hat der Weimarer Staatsrechtslehrer Hermann Heller in den 20er-Jahren des 20. Jahrhunderts eine unumstößliche Wahrheit ausgesprochen: „Es gibt keine andere Herrschaftslegitimation als die demokratische“ (Heller 1992: 309). So richtig diese Aussage noch heute ist, so klar ist auch, dass intensiveres Nachdenken über „Demokratie“ zu schwierigen Auseinandersetzungen führt. Dem kann sich die Demokratietheorie, die mit der Reflexion dieser für das Zusammenleben in unserem Gemeinwesen existenziell wichtigen Fragen beauftragt ist, kaum entziehen. Theoriebildungen über die Demokratie sind niemals nur empirische Modelle dessen, was gemeinhin für Demokratie gehalten wird. Theorien über Demokratien sollen uns auch helfen bei unseren Bemühungen, Demokratiedefizite in Herrschaftsordnungen zu erkennen und ihnen entgegenzusteuern. Wohl von kaum einer anderen Subdisziplin der Politikwissenschaft wird daher mehr erwartet, dass sie einen kritischen Stachel besitzt und - fußend auf einer möglichst exakten empirischen Problembeschreibung - normativ überzeugende Alternativen zur Weiterentwicklung demokratischer Ordnungen anbietet.

In der gegenwärtigen Debatte um legitimes Regieren jenseits des Nationalstaats beansprucht die Theorie der ,deliberativen“ Demokratie geradezu eine hegemoniale Stellung. Tatsächlich vermag sie dies nicht zu leisten, weil sie in diesem Verwendungszusammenhang unter der eigentümlichen Paradoxie leidet, sowohl überangepasst, als auch realitätsentrückt zu sein. Das spricht nicht gegen die Theorie der deliberativen Demokratie an sich, wohl aber gegen ihre exklusive Inanspruchnahme als europäische Demokratietheorie. 
Um diese These zu begründen, werde ich erstens den von mir vermuteten Paradigmenwechsel von der komplexen zur deliberativen Demokratietheorie in der Debatte um die demokratische Legitimität der EU kurz nachzeichnen. Im zweiten Schritt diskutiere ich das schwierige Verhältnis von Demokratie und Deliberation und zeige, dass nicht der Fehler gemacht werden sollte, Letztere als Surrogat der Ersteren zu konzipieren. Danach rekapituliere ich die zentralen normativen Prämissen der deliberativen Demokratietheorie und untersuche, wie diese genutzt werden, um die EU als ,deliberative Demokratie“" zu konzeptualisieren. Abschließend diskutiere ich die empirische Tragfähigkeit und normative Überzeugungskraft des deliberativen Modells in der Debatte um legitimes Regieren jenseits des Nationalstaats, indem ich am Beispiel des Kernkriteriums der ,,allgemeinen Zugänglichkeit“ einen „Lackmustest" durchführe.

\section{Paradigmenwechsel in der Debatte um legitimes Europäisches Regieren: Von der „komplexen“ zur „deliberativen“ Demokratietheorie}

Die Demokratietheorie beansprucht nicht nur, die Demokratie empirisch adäquat zu beschreiben, sondern auch in normativer Absicht sinnvolle Vorschläge zu ihrer Verbesserung machen zu können. Selbstkritisch wird indessen die politikwissenschaftliche Zunft einräumen müssen, dass sie mit ihren diesbezüglichen Forschungsbemühungen nicht immer besonders erfolgreich war. In seiner Konstanzer Antrittsvorlesung hat Fritz Scharpf bereits 1969 der politikwissenschaftlichen Demokratietheorie ins Stammbuch geschrieben, sie dürfe sich weder mit utopisch erscheinenden Demokratisierungsvorschlägen beschäftigen, weil mit diesen die politischen Realitäten kaum zum Besseren hin verändert werden könnten, noch sich zu sehr an die politischen Realitäten anpassen, um ihren kritischen Stachel nicht zu verlieren (Scharpf 1970). Damals war die demokratietheoretische Debatte tatsächlich durch diese zwei Extrempositionierungen gekennzeichnet. Eine adäquate Demokratietheorie müsste wohl - so die Intuition von Scharpf - irgendwo dazwischen liegen, und er entwickelte damals die „komplexe Demokratietheorie“, in der die normativen Anforderungen an die Demokratie mit einem sowohl empirisch-analytischen als auch mehrdimensionalen Ansatz kombiniert wurden.

Das Nachdenken über Demokratie wurde mit dieser Theorie gewiss nicht einfacher, aber man war mit ihr nicht nur in der Lage, Demokratie in all ihrer Komplexität, ihren mehrdimensionalen Legitimationsressourcen und mannig- 
faltigen Anforderungen, ihrer Vielzahl an politischen Akteuren, und schließlich ihren Herrschaftsinstrumenten und -techniken abstrakt zu beschreiben, sondern auch - von der Lincoln'schen „Gettysburg Adress“ ausgehend - konkreten Optimierungsbedarf aufzuzeigen. Allerdings hat sich die Politikwissenschaft seit jener Demokratietheorie „Zwischen Utopie und Anpassung“ nicht mehr mit einfachen Antworten auf schwierige Probleme begnügen können.

Die „komplexe Demokratietheorie“ scheint indessen manchen Demokratietheoretikern deutlich zu komplex zu sein, wenn sie über legitimes Europäisches Regieren nachdenken. Jedenfalls dominiert das Modell der deliberativen Demokratie seit etwa anderthalb Jahrzehnten die Demokratietheorie (Dryzek 2007) und erfreut sich vor allem in der Frage des demokratischen und legitimen Regierens jenseits des Nationalstaats besonderer Beliebtheit. Das Bekenntnis zu einer deliberativen Demokratietheorie fällt hier leichter, doch muss deren demokratischer Gehalt in diesem Kontext angezweifelt werden. Mehr noch: Die Theorie leidet im europäischen Verwendungszusammenhang an einer kaum aufzulösenden Paradoxie.

Einerseits passt sie sich insofern zu stark den empirisch vorfindbaren Gegebenheiten in der EU an, als sie darauf verzichtet, die Regierungspraxis in Mehrebenensystemen in ihren vielfältigen Erscheinungs- und Interaktionsformen kritisch zu hinterfragen und innovative - konkret an Institutionen ansetzende - Reformvorschläge zu machen, um der Politik so klare Handlungsoptionen aufzuzeigen. Andererseits, und das ist eigentlich noch viel ernüchternder, steht die Demokratietheorie, wenn sie im europäischen Kontext exklusiv als Theorie deliberativer Demokratie übersetzt wird, in der Gefahr, die Realitäten des Regierungshandelns komplett zu verfehlen, weil sie ihren Gegenstand, die real existierende politische Ordnung, empirisch nicht mehr als komplexes institutionelles Gefüge wahrnimmt, sondern nur noch die Ausschnitte in das Zentrum ihrer Betrachtungen stellt, von denen angenommen werden kann, dass sie den Idealvorstellungen einer deliberativen Demokratie entsprechen. Ironischerweise kommt einem dabei Hegel in den Sinn, der in Fortsetzung des aristotelisch-scholastischen Prinzips des „omne ens est verum“ einmal formuliert hat: „Was vernünftig ist, das ist wirklich; und was wirklich ist, das ist vernünftig“" (Hegel 1972: 11). Und für jene die deliberative Theorie der Demokratie besonders faszinierenden Ausschnitte gilt gleichsam in leichter deliberationstheoretischer Abwandlung: Alles was deliberativ ist, ist vernünftig. Wenn sich aber herausstellen sollte, dass die Theorie der deliberativen Demokratie mit Blick auf die Legitimationsprobleme der EU tatsächlich sowohl überangepasst als auch realitätsentrückt ist, dann stellt sich die Frage nach 
ihrem wirklichen Nutzen in aller Schärfe. Dies gilt es im Folgenden näher zu überprüfen.

\section{Deliberation statt Demokratie? Zur Anwendung der Theorie der deliberativen Demokratie im europäischen Kontext}

Deliberation statt Demokratie - so könnte man das Credo vieler neuerer demokratietheoretischer Abhandlungen umreißen, wenn diese das Regieren jenseits des Nationalstaats thematisieren: Aus der Verlegenheit heraus, vor allem die angesichts der internationalen und europäischen Rechtsetzungs- und Verrechtlichungsprozesse immer drängender werdende Legitimitätsfrage des Regierens jenseits des Nationalstaats auf traditionelle Weise nicht lösen zu können, werden voluntaristisch die generellen Legitimitätsanforderungen heruntergeschraubt. Was dann an Legitimationsbedarf übrig bleibt, sollte möglichst nicht mehr durch auf Einstimmigkeit beruhende intergouvernementale Verhandlungssysteme oder aber durch altbekannte parlamentarisch-demokratische Legitimationsmuster gedeckt werden. Vielmehr sollten an ihre Stelle möglichst post-parlamentarische und expertenbasierte Deliberation treten.

Hat man früher aufgrund der Zunahme an Komplexität und Steuerungsproblemen mit der komplexen Demokratietheorie reagiert, macht man es sich heute in der Demokratietheorie - so scheint es - gerne einfacher, wenn die Chancen demokratischen Regierens in der EU erörtert werden: Expertenbasierte und deliberative Entscheidungsfindung - egal ob in der Komitologie (Joerges/ Neyer 1997), in der nationalen und supranationalen Gerichtsbarkeit (HitzelCassagnes 2004), in „governance networks“ (Sørensen/Torfing 2005) oder in (deliberativen) Diplomatenrunden (Müller 1994) - genießt inzwischen fast schon einen Legitimationsüberschuss, gerade weil sie demokratisch defizitär ist. Gerade weil und insoweit deliberative Entscheidungsfinder nicht mit sie einschränkenden Konstituenten demokratisch rückgekoppelt sind und insofern auch keine besondere Rücksicht auf Einzelinteressen mehr nehmen müssen, unterwerfen sie sich alle der postulierten Rationalität der Deliberation, durch die Entscheidungen problemlösungsorientiert getroffen werden können. In derartigen Diskursarenen kann dann auch getrost auf herkömmliche demokratische Muster der Entscheidungsfindung verzichtet werden.

Auf elegante Art wird so vermieden, dass die Kopplung zwischen denen, die herrschen, d. h. kollektiv verbindliche Entscheidungen treffen, und denen, die von diesen jeweiligen Entscheidungen in ganz unterschiedlicher Weise konkret betroffen sind, zu eng wird. Bei zu enger Kopplung, das wissen wir aus 
der Organisationssoziologie, fehlt den Entscheidungsträgern aufgrund ihres dann imperativ wirkenden Mandats aber die Diskurs- und Argumentationsfreiheit, mithin der Spielraum, um miteinander problemlösungsorientiert argumentieren und deliberieren zu können. Entscheidungsfindung sollte also möglichst auf Deliberation, auf Beratung basieren. Dabei wird aber ein zentrales Problem übersehen: Da die Vorzüge der Deliberation nicht im Kontext einer auf Repräsentation beruhenden freiheitlich-demokratischen Ordnung diskutiert werden, führt es zu einer gewissen theoretischen Nähe zum aufgeklärten Absolutismus, wenn man für Konzepte demokratischen und legitimen Regierens in der EU plädiert, die auf das Moment der Deliberation beschränkt sind. Auch ein aufgeklärter Monarch ist zur Aufrechterhaltung seiner Herrschaft ja gut beraten, sich gut beraten zu lassen.

An diesem Punkt wird das Verhältnis zwischen „Deliberation“ und „Demokratie" jedoch sehr problematisch, weil beide Begriffe keineswegs das Gleiche bedeuten. Zwischen ihnen kann sogar ein Spannungsverhältnis bestehen. In einer funktionsfähigen verfassungsstaatlich organisierten Demokratie koexistieren repräsentativ-demokratische und deliberative Momente, beide haben dort in der historischen Entwicklung demokratischer Verfassungsstaaten ihren legitimen Ort gefunden. Und dennoch müssen beide in einem ausgewogenen Verhältnis zueinander stehen; so darf z. B. das deliberative Moment in der Verfassungsgerichtsbarkeit nicht einseitig auf Kosten des repräsentativ-demokratischen Moments, wie wir es im parlamentarischen Gesetzgeber vorfinden, gehen. Beides, Deliberativität und Demokratizität, macht gemeinsam die Legitimität des demokratischen Verfassungsstaates aus. Wer aber das in der Entwicklung moderner demokratischer Verfassungsstaatlichkeit angelegte Spannungsverhältnis von Deliberation und Demokratie übersieht, kann Deliberation sonst sehr leicht als (normativ mindestens ebenso anerkennungswürdiges) Surrogat für nicht existierende Demokratie konzeptualisieren - und dieses dann aber in äußerst missverständlicher Manier als „,deliberative Demokratie“ verkaufen.

Übersehen wird dabei, dass Demokratie und Deliberation in bestimmten Konstellationen - gerade in der postnationalen Konstellation - auch miteinander im Konflikt liegen können und Letzteres Ersteres nicht einfach zu ersetzen vermag. Das zentrale normative Problem, das bisher übersehen wird, ist daher Folgendes: Wenn man der Theorie der deliberativen Demokratie abnimmt, dass expertokratisch-deliberative Entscheidungsnetzwerke aufgrund ihres problemlösungsorientierten kollektiven Entscheidungsstils überlegen und normativ vorzuziehen sind - könnte man dann nicht getrost auf demokratische Rückkopplung verzichten? Deliberationstheoretisch konsequent zu Ende gedacht vielleicht 
sogar dort, wo diese demokratische Rückkopplung bei allen zu konstatierenden Schwierigkeiten noch einigermaßen funktioniert, nämlich im parlamentarischdemokratischen Verfassungsstaat, in dem jede staatliche Gewalt zumindest dem demokratischen Anspruch nach ,vom Volke“ ausgehen soll und über Wahlen legitimiert wird (kritisch hierzu Nullmeier 2008)? Anders - und in polemischer Zuspitzung gefragt: Wie weit soll denn die Vernunft der Deliberation (auf Kosten der Demokratie) gehen? So weit, dass traditionelle, auf dem Prinzip der Volkssouveränität basierende Herrschaftsordnungen mit all ihren Rückkopplungsmechanismen und Legitimationsketten (Böckenförde 1987) völlig diskreditiert werden? Betrachtet man die Debatte um die Legitimität des Regierens jenseits des Nationalstaats genauer, kann man jedenfalls durchaus den Eindruck gewinnen, dass „Momente von Deliberativität und Demokratizität auseinandertreten und sich zu rivalisierenden Legitimitätskonzepten zu verfestigen scheinen" (Niesen 2007: 1). Stattdessen müsste eine wahrhaft europäische Demokratietheorie versuchen, die in der europäischen Governance-Praxis tatsächlich auseinanderdriftenden Momente als zusammengehörige Seiten einer Medaille wieder zusammenzudenken. Zweitens müsste sie anhand von Vorschlägen, die konkret an Institutionen ansetzen, zeigen, wie das deliberative und das demokratische Moment gleichzeitig gestärkt werden könnte - (ähnlich: Sonnicksen o. J.).

\section{Die EU als ,deliberative“ Demokratie?}

Was sind die zentralen Aussagen der Theorie der deliberativen Demokratie im europäischen Verwendungszusammenhang, auf welchen Prämissen baut sie auf, welche sind ihre normativen Implikationen und wie finden diese Anwendung bei Modellen des legitimen Regierens jenseits des Nationalstaats? Die Definitionen von Demokratie in deliberationstheoretischen Schriften sind höchst anspruchsvoll: ${ }^{1}$ Der gemeinsame Nenner ist, dass Demokratie nicht weniger als eine gemeinschaftliche Autorschaft der Gesetze sicherstellen müsse. Selbstgesetzgebung als Ausdruck kollektiver politischer Autonomie heißt das Zauberwort. Um dem anspruchsvoll daherkommenden Bild einer idealen Demokratie zu entsprechen, muss nach Joshua Cohen das politische System einer deliberativen Demokratie der Anforderung genügen, dass die Ergebnisse politischer Entscheidungsfindung das Ergebnis eines freien und begründeten Einverständnisses unter Gleichen sind (Cohen 1989: 22).

1 Vgl. zum Folgenden die Beiträge der wichtigsten Vertreter der Theorie in Bohman 1997; Elster 1998; Habermas 1992. 
Damit klingt an, dass die deliberative Demokratie - und dies macht wohl vor allem ihre Attraktivität aus - sowohl rationale als auch partizipative Aspekte hinreichend berücksichtigt. Das Einverständnis, das zu einer Entscheidung führt, muss sowohl begründet sein als auch auf der gleichen Teilhabe freier Bürger und der Inklusion aller ihrer Präferenzen, Einwände und Argumente beruhen, die allesamt in den politischen Prozess eingespeist werden müssen. Letztlich geht es also nur durch Einbeziehung aller von einer Entscheidung Betroffenen (Benhabib 1996: 69). Das klingt überzeugend und sehr demokratisch - doch kann es in der Praxis funktionieren? Und was nutzt ein solches Demokratieverständnis im Hinblick auf die Legitimation einer komplexen überstaatlichen Herrschaftsordnung, wie wir sie in der EU zweifellos vorfinden (Eriksen 2005)?

Zentral für die deliberative Demokratietheorie ist, dass sie die von Fritz Scharpf eingeführte Unterscheidung zwischen Input- und Output-Legitimation gewissermaßen transzendieren will, indem sie diese beiden Momente wieder zusammenführt, was aber - wie noch zu zeigen sein wird - letztlich auf Kosten des „Input“ geht. Scharpf hat in seiner „komplexen Demokratietheorie“ zwar immer die Auffassung vertreten, das Output, also die Herrschaft für das Volk, entscheidend wichtige Leistungen zu erbringen imstande sei, doch auf Input, Herrschaft durch das Volk, letztlich nicht verzichtet werden könne, um von einer demokratischen politischen Ordnung zu sprechen. Eine legitimationstheoretisch sinnvolle Evaluation einer Demokratie darf sich daher nicht darauf beschränken, nur nach der Qualität von Politikergebnissen zu fragen. Dies wäre aus zwei Gründen außerordentlich fragwürdig: Erstens bliebe dann unklar, wer denn zur Beurteilung berufen ist, ob ein Politikergebnis eine gute Qualität aufweist oder nicht, und zweitens lässt sich ein politisches System kaum als demokratisch kennzeichnen, wenn die Konzeption einer Herrschaft für das Volk und mit ihr die Reduktion der Demokratietheorie auf Politikergebnisevaluation sich kaum mehr von autokratischen und damit nicht-demokratischen Systemen unterscheidet. Insofern hat Claus Offe doch recht, wenn er betont, dass nicht die „Politikergebnisse demokratisch sind, sondern der Modus ihres Zustandekommens" (Offe 2005: 264). Scharpf mag in seinen demokratietheoretischen Analysen zur EU aufgrund ihrer mangelnden Demokratiefähigkeit sein Hauptaugenmerk auf die Output-Dimension gelegt haben, doch wäre er kaum auf die Idee gekommen, mittels dieser Akzentuierung ,die fortschreitende Entdemokratisierung Europas demokratisch zu bemänteln" (so aber der Vorwurf bei Brunkhorst 2003: 377). Er hat dem Demokratiedefizit der EU einen viel zu 
prominenten Stellenwert eingeräumt, als dass ihm diese Unterstellung ernsthaft gemacht werden könnte.

Diesen letzten Schritt - die demokratische Bemäntelung der Entdemokratisierung - hat dann erst die Theorie der deliberativen Demokratie selbst getan. ${ }^{2}$ Für sie ist die EU eine „deliberative Demokratie“, zumindest ein Gebilde, das sich energisch dazu entwickelt und auch weiter entwickeln sollte. Die deliberative Demokratie in Europa wird aus dieser Sicht dadurch realisiert, dass deliberative Verfahren in Form von Kommunikationsprozessen, bei denen das beste Argument zählt, zunehmend an die Stelle strategischen Verhandelns und lediglich funktional indizierter Anpassungsprozesse tritt. Zu einer solchen Sichtweise, die in der Identifikation von gemeinschaftlichen Lern- und Argumentationsprozessen zugleich die Essenz von Demokratie schlechthin zu erkennen glaubt, kann man jedoch nur kommen, wenn man den Demokratiebegriff konsequent „semantisch verschiebt“ (Buchstein/Jörke 2003: 471) und ihn seines traditionellen Input-orientierten Bedeutungsinhalts beraubt.

Man kann dies wie Hubertus Buchstein und Dirk Jörke durchaus als fragwürdiges Ergebnis einer „Rationalisierung der Demokratietheorie“ (2003: 474) begreifen, die ihren Fokus auf die Bewertung der Qualität von Politikergebnissen richtet (Output), während traditionelle Vorstellungen zur Input-Legitimation allmählich an Bedeutung verlieren. Daher ist es nur folgerichtig, dass die Institutionen, von denen allgemein angenommen wird, dass sie jene klassische Input-Legitimation zu leisten imstande sind, kaum mehr in den Blick genommen werden. In deliberativ-demokratischen Ansätzen zur EU tauchen konsequenterweise das Europäische Parlament und mit ihm verbunden eine fundierte Debatte um allgemeine, freie und gleiche Wahlen sowie Reflexionen zur demokratischen Rückkopplung europäischer Herrschaftsausübung mit einem souveränen Wählerwillen kaum mehr auf. Selbst minimale Mechanismen einer demokratischen Herrschaftskontrolle gegenüber der europäischen Exekutive scheinen kaum mehr eine Rolle zu spielen. ${ }^{3}$

An ihrer Stelle findet eine sowohl überangepasste als auch die Realitäten aus dem Blick verlierende Auseinandersetzung mit dem Legitimationsproblem des Europäischen Regierens statt. Überangepasst, weil das real existierende und real auch mit diversen Demokratiedefiziten gekennzeichnete politische

2 Gerstenberg/Sabel 2001; Schmalz-Bruns 2002; Neyer 2006, 2007.

3 Es entspricht dem Weltbild der an diversen diskursiven Modellen globaler Demokratie werkelnden Deliberationstheoretiker, dass Legitimation durch Wahlen zunehmend irrelevant wird und daher zivilgesellschaftliche Diskurse national, supranational und transnational in die Bresche springen müssten (Dryzek 2006). 
System indirekt als sakrosankt erklärt wird, zumal es mit klassischer institutioneller Reformpolitik nicht demokratisierbar sei. ${ }^{4}$ Realitätsentrückt, weil eine solche Demokratietheorie ihren eigentlichen Gegenstand - nämlich die Ermöglichungsbedingungen von echter Demokratie in einer überstaatlichen Herrschaftsordnung - zunehmend aus den Augen verliert, indem sie die aus einem eher klassischen Verständnis von Demokratie herrührenden und empirisch feststellbaren Zweifel an der demokratischen Qualität der europäischen Regierungspraxis nicht mehr zur Kenntnis nehmen will.

Fragwürdiger noch ist, dass die von einer herkömmlichen demokratietheoretischen Warte durchaus als problembehaftet zu bezeichnende Praxis europäischen Regierens (Follesdal/Hix 2005; Höreth 1999) von der deliberativen Demokratietheorie normativ einfach umgedeutet wird. Hier findet eine gewissermaßen nietzscheanische „Umwertung aller Werte“ statt, indem demokratietheoretische Probleme umstandslos zu deliberativen Ideallösungen stilisiert werden: eine sich der parlamentarischen Kontrolle weitgehend entziehende Exekutivund Elitenkartellisierung sowie die mit ihr verbundene Technokratisierung kollektiver politischer Entscheidungsfindung unter Ausschluss einer europäischen Öffentlichkeit und effektiver Herrschaftskontrolle „von unten“. Das exekutivlastige Regieren ohne nennenswerten parlamentarischen Input mündet nach dieser Interpretation in einen deliberativen Supranationalismus einer postparlamentarischen Demokratie, in der die „List der Vernunft“ obsiegen wird, weil am Ende alle politischen Entscheidungsträger dann doch gut beraten sind, sich durch das bessere Argument überzeugen zu lassen.

Vor diesem Hintergrund ist es aus demokratietheoretischer Sicht nur ein schwacher Trost, dass sich in der europäischen Politik funktional differenzierte Teilöffentlichkeiten bilden, die sich durch gemeinsame Interessen konstituieren und als dezentrale und grenzüberschreitende Problemlösungsgemeinschaften wirken, deren Interaktionen keine nationale Schicksalsgemeinschaft mehr voraussetzen (Bohman 2005). Die so möglich werdenden nicht-hierarchischen Formen politischer Steuerung, die für das Europäische Regierungssystem so eigentümlich sind, können keineswegs als entscheidende Merkmale für die Demokratizität des europäischen Governance-Systems betrachtet werden,

4 In diesem Sinne überangepasst ist im Übrigen auch die aus einer - hier nicht weiter zu untersuchenden - „realistischen“ Perspektive aufgestellte Behauptung, das Beharren der Demokratietheorie auf vermeintlich überkommenen Vorstellungen von Partizipation sei mit Blick auf die EU irrational, weil supranationales Regieren zum einen relativ wenig politische Partizipation erforderlich mache, zum anderen die EU ein den nationalen Demokratien bereits durchaus vergleichbares Legitimitätsniveau erreicht habe (Moravcsik 2004: 362). 
sondern allenfalls als Foren vorhandener Deliberativität (Risse 2004). Essenziell für die Demokratie ist hingegen noch immer, dass generell ein wirksamer Bindungsmechanismus zwischen dem Entscheidungssystem und den Ansprüchen der Bürgerschaft bestehen muss - auch zu jenen Bürgern, die zivilgesellschaftlich bedauerlicherweise nicht organisiert und wohl auch keine Mitglieder jener „funktional differenzierten“ Teilöffentlichkeiten sind. Durch diverse funktionale Demoi kann jedenfalls das Prinzip der demokratischen Selbstbestimmung kaum verwirklicht werden, wenn den herkömmlichen Demoi im Zweifel nur die Zuschauerrolle und ansonsten buchstäblich „keine Wahl“" mehr bleibt.

Dieser Kritik könnte man freilich entgegnen, dass die deliberative Demokratietheorie doch immerhin zwischen einer Input- und einer Output-Dimension zu vermitteln versucht (so z. B. Niesen 2007: 6). Auf Partizipation, also auf Input, muss es ihr doch schließlich auch ,irgendwie“ ankommen, denn von alleine können die geforderten „guten Politikergebnisse“ ja nicht zustande kommen. ${ }^{5}$ Zwischen Input und Output muss in einer Prozessdimension vermittelt werden - das Verfahren des Zustandekommens von Ergebnissen, der sogenannte ,through-put“", übernimmt daher selbstverständlich eine legitimierende Funktion. Auch von der deliberativen Demokratietheorie würde man dazu gerne Konkreteres erfahren: über die zu einer Entscheidung berufenen Akteure, ihre Präferenzen, ihre institutionellen und parteilichen Eigeninteressen und ihre demokratische Rückkopplung, die generellen Informations- und Machtasymmetrien im politischen System, über die verschiedenen, von den unterschiedlichen Akteuren aus unterschiedlichsten Gründen präferierten Interaktionsmodi, den jeweiligen Konfliktgehalt der zur „Beratung“ stehenden Entscheidungsgegenstände - um nur eine wenige interessante Aspekte des ,through-put“ zu nennen, der am Ende zu einem Ergebnis führen soll.

Mehr als ein ehernes Bekenntnis zur „partizipativen“ Demokratie, die den Prozess kennzeichnen soll, an dessen Ende ein den deliberativen Grundsätzen entsprechende Entscheidung stehen soll, ist den meisten theoretischen Abhandlungen jedoch nicht zu entnehmen, weil sie in seltsamer Weise ,institutionenneutral" argumentieren, so als ob sich die Vernunft geradezu unabhängig von Institutionen immer Bahn brechen könnte, wenn denn nun endlich von allen Akteuren die Vernünftigkeit der Annahmen der deliberativen Demokratietheorie erkannt und auch anerkannt würde. Dazu bedarf es im Übrigen anscheinend nur einer möglichst ,aktiven Zivilgesellschaft“, die sich wirkungsmächtig Gehör und Einfluss zu verschaffen vermag - wenngleich nie ganz klar wird, wer denn nun zu dieser aktiven und partizipierenden Zivilgesellschaft gehört. Das

5 Radikal auf umfangreiche Partizipation setzt etwa Benhabib 1996. 
für die Demokratie so zentrale Problem der Inklusion und Exklusion bleibt mithin ungelöst (Young 2001a).

Was kann unter diesen Bedingungen das hehre Bekenntnis zu „Partizipation“ eigentlich bedeuten? Es bedeutet zunächst, auf einer theoretischen Ebene, dass nicht berücksichtigte Positionen, Perspektiven und Argumente der am Ende stehenden Entscheidung die Legitimität entziehen, ergo Partizipation dringend notwendig ist. Entscheidender ist hingegen ein anderer Gesichtspunkt: Ohne Beteiligung oder zumindest Nachvollzug des politischen Prozesses kann sich eine legitimierende Kraft „dort, wo es darauf ankommt, nämlich in gesellschaftlichen Assoziationen und letztlich in den Köpfen der Bürger nicht entfalten“ (Niesen 2007: 9).

Doch wie ist es tatsächlich um die so gerne betonte Partizipation innerhalb des Modells der deliberativen Demokratie bestellt? Die Durchsicht verschiedener deliberationstheoretischer Modelle hinterlässt diesbezüglich einen zwiespältigen Eindruck. Einerseits betont etwa Rainer Schmalz-Bruns, dass die „der Deliberation zugeschriebenen, zivilisierenden Wirkungen [...] sich [...] nur unter Bedingungen unmittelbarer Teilhabe einstellen [können] und nicht etwa dadurch, dass man die Prüfung der Rechtfertigbarkeit von Ergebnissen separaten, paternalistischen Institutionen überträgt“ (1999: 218). Insoweit hier mit dem Hinweis auf Teilhabe das partizipative Moment angesprochen wird, darf man sich diese jedoch nicht als einen Willensbildungsakt von „unten nach oben" vorstellen, wie einer der Urväter der deliberativen Demokratie, Jürgen Habermas, betont. Er insistiert stattdessen darauf, dass das demokratische Verfahren ,seine legitimierende Kraft nicht mehr nur, und nicht einmal in erster Linie, aus Partizipation und Willensäußerung [bezieht], sondern aus der allgemeinen Zugänglichkeit eines deliberativen Prozesses, dessen Beschaffenheit die Erwartung auf rationale Ergebnisse begünstigt" (Habermas 1998: 166).

Was genau ist hier mit der Demokratie passiert? Man kommt der Sache auf den Grund, wenn diese normativen Ansichten zunächst damit konfrontiert werden, was ich als das „Versprechen“ der Demokratie bezeichne. Dabei stellt sich ein normatives Problem: Habermas' Zitat lässt sich dahingehend interpretieren, dass Deliberation und Demokratie, wie wir sie herkömmlich verstehen, auseinanderfallen können, denn ,allgemeine Zugänglichkeit“ und präsumtive Rationalität sind etwas anderes als Selbstgesetzgebung. Wenn eine Demokratie von Wert sein will, muss sie aber ihrer Bestimmung, ihrer ,raison d'être“, gerecht werden: In der Demokratie muss der Demos dem Wortsinne entsprechend herrschen (kratia). 
Eigentlich enthält der Rekurs auf die Demokratie daher immer ein Versprechen auf politische Willensbildung von unten nach oben und weckt Erwartungen, die sich zuvörderst auf die partizipative Komponente des Demokratieideals beziehen (vgl. die brillante Beweisführung bei Buchstein/Jörke 2003: 482). Natürlich ist dieses Versprechen eine regulative Idee, die immer nur unvollständig verwirklicht werden kann, doch die für die demokratische Qualität eines Gemeinwesens entscheidende Frage ist noch immer, ob ihre Bürger in hinreichendem Umfang an der Gesetzgebung beteiligt werden (Niesen 2007: 6). Von diesem Postulat rückt Habermas aber bei Lichte besehen geradezu radikal ab, wenn er zwar die deliberative Demokratie als die beste aller möglichen Regierungsformen favorisiert, sich dies aber nur durch die Klassifizierung der politischen Partizipation als zweitrangig leisten kann. Er kann die von ihm postulierte Deliberation mit der Demokratie also nur dadurch versöhnen, dass er den Demokratiebegriff systematisch ausdünnt und hierbei insbesondere die ihm in den 70er-Jahren noch so heilige Komponente der politischen Beteiligung den Funktionalitäts- und Rationalitätszumutungen seiner deliberativen Demokratie unterordnet (dazu Jörke 2003: 222 ff.). An die Stelle der Demokratie setzt Habermas daher die „Deliberatur“. Simone Chambers hat dies auf eine griffige Formel gebracht: „Talk-centric democratic theory replaces voting-centric democratic theory." (2003: 304) Es ist rezeptionsgeschichtlich wirklich erstaunlich, dass ausgerechnet dies immer wieder übersehen wird, doch um Partizipation geht es Habermas und anderen Vertretern der Theorie der deliberativen Demokratie gerade nicht mehr, ${ }^{6}$ sondern um allgemeine Zugänglichkeit.

Kontrastieren wir nun die oben zitierte Schlüsselaussage von Habermas zur legitimierenden Kraft von demokratischen Verfahren mit den empirischen Fakten des europäischen Regierens, dann müsste, was die politische Partizipation betrifft, mit Habermas festgestellt werden, dass es auch der EU nicht in erster Linie um Partizipation geht und sie somit dieses erste - negative - Kriterium bestens erfüllt. Doch wie sieht es um die von Habermas postulierte allgemeine Zugänglichkeit deliberativer Prozesse in Europa aus? Was bedeutet dies in trans- und supranationalen Kontexten? Als gegebene Selbstverständlichkeit europäischer Regierungspraxis kann sie kaum angesehen werden. Es gilt daher noch etwas genauer zu untersuchen, was im Rahmen der EU mit ,allgemeiner Zugänglichkeit eines deliberativen Prozesses“ überhaupt gemeint sein kann.

6 Deutlich wird dies etwa bei der Behandlung der partizipativen und repräsentativen Komponente deliberativer Politik durch Parkinson 2003. 
Schließlich ist sie die Voraussetzung dafür, dass rationale Ergebnisse erwartet werden können.

\section{Lackmustest: Zur „allgemeinen Zugänglichkeit“6 deliberativer Prozesse in der $\mathbf{E U}$}

Wenn eine Theorie von Wert sein will, dann muss sie auch die Zumutung über sich ergehen lassen, mit Fakten konfrontiert zu werden. Das gilt auch für die Habermas'sche „Deliberatur“, die in der Lesart mancher EU-Forscher unter dem missverständlichen Etikett einer Demokratietheorie daherkommt: Wo und bei wem wäre denn mit Blick auf die EU eine demokratietheoretische Unterstellung plausibel, man könne allgemein zugängliche deliberative Prozesse beobachten, die aufgrund ihrer Beschaffenheit bei uns die Erwartung auslösen, rationale Ergebnisse zu produzieren?

Hier stößt man auf ein ganz seltsames Phänomen. Während manche die EU insgesamt zuweilen als System des „deliberativen Supranationalismus“ (Schmalz-Bruns 1999) preisen, wird bei genauerer Betrachtung lediglich - und ausgerechnet - anonymen Expertengremien, die ein Schattendasein in der europäischen Politik fristen, jene normativ vorzugswürdige deliberative Qualität attestiert. Diese können jedoch kaum als ,allgemein zugänglich“ bezeichnet werden und wer keinen Zugang hat, wird auch nicht beurteilen können, ob man von jenem angeblich deliberativen Prozess rationale Ergebnisse erwarten darf. Das können in sinnvoller Weise nur Menschen mit Zugang beurteilen, also jene mit ,insider's wisdom“ (Shapiro 1999a: 80) ausgestatteten Privilegierten, die Teil des deliberativen Entscheidungsnetzwerkes selbst sind. Diese werden in der Absicht einer selbstreferenziellen Legitimation ihr Treiben aber naturgemäß genauso positiv beurteilen wie dies Mitglieder einer herkömmlichen Staatsregierung mit Blick auf ihre Entscheidungen tun würden.

Der gravierende - und die Funktionsfähigkeit einer Demokratie ausmachende - Unterschied ist jedoch, dass sich eine Staatsregierung verantworten muss: gegenüber ihren Wählern, gegenüber dem Parlament, gegenüber den betroffenen Interessengruppen, gegenüber einer sie kritisch beäugenden Öffentlichkeit. Und wenn man mit den Policy-Outputs jener Regierung nicht einverstanden ist, dann straft man sie im Schumpeter'schen Sinne bei den nächsten Wahlen ab und lässt die „Anderen“ ran. Grundbedingung hierfür ist indessen ausgerechnet jene ,Öffentlichkeit", derer die Deliberation gerade nicht bedarf: „Indeed, the principled cooperation and search for common ground characteristic 
of authentic deliberation is [...] to be undermined by publicity." (Shapiro 2002: 198)

Dass die Deliberationsnetzwerker auch aufgrund der Absenz einer sie kritisch beäugenden Öffentlichkeit ihre „Abwahl“ bzw. Ersetzung nicht befürchten müssen, ist aus Sicht der Deliberationstheoretiker kein demokratietheoretisches Übel, sondern ein legitimationstheoretischer Mehrwert. So werden eigentlich unerwünschte Erscheinungen zum Vorteil: die fehlende demokratische Rückkopplung, die lose Kopplung, die nicht bestehende Bindung der Experten an irgendeinen Wählerwillen, die fehlende Beauftragung durch das Volk, die nicht bestehende Angst, bei falschen Entscheidungen bei den nächsten Wahlen politisch zur Verantwortung gezogen und abgewählt zu werden, ja sogar das schlichte Desinteresse - oder, wie es John Fossum einmal schön formuliert hat, das „ohrenbetäubende Schweigen“ (Fossum/Trenz 2006) - weiter Teile der EUBevölkerung in EU-Angelegenheiten. Sie sind insofern Voraussetzungen ,ex negativo" für die Qualität der Deliberation als ihr eigentlich erwünschtes Gegenteil die Teilnehmer der Deliberation nur diskursiv belasten würde. Gerade die Leistungsfähigkeit der oft als Nukleus einer entstehenden deliberativen Demokratie in Europa angeführten Komitologie lässt sich ja auf die weitreichende Abkopplung der in ihr stattfindenden Expertendeliberation von direkter partizipatorischer Einflussnahme zurückführen, was im Übrigen von den Deliberationstheoretikern auch eingeräumt wird (Joerges/Neyer 1977: 222).

Um es noch deutlicher zu formulieren: Nur wenn man mit keinen negativen Sanktionen seitens irgendwelcher Konstituenten rechnen muss und sich insofern unter Freien und Gleichen gemeinsam um eine beste Lösung bemüht, stellt sich jene vorbehaltlose und problemlösungsorientierte Offenheit ein, derer die Deliberation bedarf. So gesehen präsentiert sich die Demokratie in einem geradezu dramatisch schlechten Licht, denn in ihr können Akteure nur selektiv und differentiell auf Argumentationsressourcen zurückgreifen, um ihre Position etwa als Regierungsmitglied, Fraktionsvorsitzender oder Oppositionsmitglied nicht zu gefährden. Sie sind zu eng an ihre Konstituenten, etwa an den Willen ihrer Wähler, gekoppelt. Eine Offenheit gegenüber allen möglichen Argumenten, oder, wie es im Jargon der Deliberationstheoretiker heißt, ein ,uneingeschränkter Zugriff auf alle zur Verfügung stehenden Gründe“, die ein zu entscheidender Fall aufwirft, kann hier kaum unterstellt werden.

Wie schon angedeutet, darf man dies aus Sicht der Deliberationstheoretiker bei Expertengremien in der EU aber durchaus. In den diversen EU-Ausschüssen der Komitologie arbeiten Akteure sach- und problemlösungsorientiert 
zusammen, verstricken sich kontinuierlich in Beratungen, in denen sich zumeist das beste Argument durchsetzen kann, gerade weil es den Mitgliedern dieser Ausschüsse gelingt, sich von ihrer Funktion als nationale Interessenvertreter abzukoppeln (Joerges/Neyer 1998). Das ist alles durchaus zu begrüßen - doch kann man aus dieser Perspektive bereits die lose Kopplung als demokratisches Allheilmittel anpreisen?

Man darf annehmen, dass diese Beschreibung expertokratischer Entscheidungsprozesse in der EU auf richtigen Beobachtungen basiert, doch stellen sich gleich mehrere normative Probleme. Das zentrale Problem lässt sich wie folgt auf den Punkt bringen: Demokratie ist Kopplung - Deliberation Entkopplung. Dies hat zur Folge, dass sich Deliberation, so wie sie ihre Anhänger verstanden wissen wollen, und Demokratie, wie es einem herkömmlichen Verständnis entspricht, wechselseitig ausschließen können. Es ist geradezu das Signum moderner Demokratie, dass in ihr staatliche Gewalt durch einen wirksamen Bindungsmechanismus zwischen dem Entscheidungssystem und den Ansprüchen der Bürgerschaft gebändigt wird: Bindung an, Beauftragung durch und Rückkopplung mit ihren Konstituenten - z. B. den Wählern - ist für die zu kollektiv verbindlichen Entscheidungen berufenen politischen Akteure in einer Demokratie prinzipiell unverzichtbar. Ausgerechnet diese Bindungen sind aber auch das Gift, das vorbehaltlose, problemlösungsorientierte und letztlich deliberative Interaktionen zwischen Akteuren in der Wettbewerbsdemokratie, die unterschiedliche Interessen und Wählerschichten repräsentieren, oft unmöglich macht. Insofern gilt für die EU folgende Umkehrung der Werte, die freilich so nie offen ausgesprochen wird: Je mehr Deliberation, desto besser, auch wenn dies weniger Demokratie im konventionellen Sinne bedeutet. Umgekehrt gilt: Je weniger Demokratie auf EU-Ebene, desto mehr Spielraum für Deliberation, weil dann immer mehr Akteure sich von diskursiven Belastungen befreien können, unter denen politische Mandatsträger so leiden müssen. Umso uneingeschränkter ist dann auch der Zugriff auf potenziell alle zur Verfügung stehenden Argumente, um dann auf dieser Grundlage miteinander kommunikativ zu handeln und „richtige“ Lösungen zu suchen. Aber findet Deliberation in der Realität tatsächlich so statt, wie es den Vorgaben ihrer Theorie entspricht? Und ist das eine normativ vorzugswürdige Alternative zur repräsentativen Demokratie, wie wir sie herkömmlich verstehen und praktizieren, so wie sie sicher auch vom eingangs zitierten Hermann Heller verstanden wird, wenn er bekennt: „es gibt keine andere Herrschaftslegitimation als die demokratische“? Sollte man tatsächlich den Teufel des europäischen Demokratiedefizits mit dem Beelzebub des „deliberativen Supranationalismus“ austreiben? Oder 
handelt man sich mit der erfolgreichen deliberativen Bekämpfung der Pest des Demokratiedefizits nur die Cholera einer zwar aufgeklärten, aber doch vordemokratischen Herrschaftsform ein?

Um das herauszufinden, müssen wir noch ein letztes Mal zurück zur vorhin zitierten Definition von Habermas. Die ,allgemeine Zugänglichkeit eines deliberativen Prozesses", von der er spricht, ist in der EU offensichtlich nirgendwo gegeben. Inklusion ist nur vermeintlich, nicht aber wirklich die Stärke der deliberativen Entscheidungsnetzwerke in der EU, denn wer deliberieren will, muss Experte sein, bedarf der Ressourcen Zeit, Wissen, auch Macht und Einfluss und muss wohl Mitglied der Zivilgesellschaft sein (Sirianni 2001). Zudem darf die Anzahl der miteinander Deliberierenden nicht zu groß sein: „It is true that genuine deliberation can happen only in small-scale forums" (Parkinson 2003: 191). Das schließt viele Menschen von vornherein aus - von einer ,allgemeinen" Zugänglichkeit kann also kaum die Rede sein.

Nun könnte man sich aber über diese Tatsache hinwegtrösten, wenn und insoweit die Teilnehmer der Deliberation die Nichteilnehmenden als Trustees repräsentierten. Wenn aber die Auswahl der Deliberationsteilnehmer und Mitentscheider auf dem Modus expertokratischer Selektion statt auf demokratischer Elektion beruht, dann ist die geforderte ,allgemeine“ Zugänglichkeit für die von der Deliberation faktisch Ausgeschlossenen jedoch nicht gegeben. Wäre es dann nicht ehrlicher und insofern auch „empirischer“, von einer ,speziellen“ bzw. „selektiven“ Zugänglichkeit für „Teilöffentlichkeiten“7 zu sprechen, da zur Aufrechterhaltung der Deliberativität entsprechender Foren die Inklusion der qualifizierten Wenigen immer mit einer Exklusion der weniger oder gar nicht qualifizierten Vielen einhergehen muss?

Deliberation setzt überdies voraus, dass die Teilnehmer rational und problemlösungsorientiert argumentieren und nicht interessenbezogen egoistisch. Doch wenn wir von echten Menschen sprechen, die politische Entscheidungen zu treffen haben, dann müssen wir annehmen, dass sie alle ebenso echte Interessen haben, die sich übrigens auch voneinander unterscheiden können (Shapiro 1999b). Die Teilnehmer an einer Deliberation könnten beispielsweise das

7 Da es sich bei der ,allgemeinen Zugänglichkeit“ eigentlich um eine hochselektive Zugänglichkeit handelt, stellt sich erstens die Frage, ob dies nicht doch auch (europäischer) Ausdruck des schon in den 60er-Jahren von Habermas konstatierten „Strukturwandels der Öffentlichkeit“ (1962) sein könnte. Und zweitens kommt der Verdacht auf, dass ausgerechnet die von Habermas in seinem Werk ,Legitimationsprobleme im Spätkapitalismus“ (1973) kritisierte Ersatzfunktion von Politik und deren scheindemokratische Prozeduren sowie die später diagnostizierte Entkopplung der Lebenswelten im deliberativen Supranationalismus fröhliche Urständ feiern. 
Interesse haben, das nächste Mal wieder in das betreffende Gremium eingeladen zu werden. Um nicht allzu negativ aufzufallen, vermeiden sie eventuell sachlich durchaus begründete Widerworte gegen eine Argumentation, von der sie annehmen müssen, dass mit ihr eine Auffassung vertreten wird, die von der großen Mehrheit geteilt wird, obwohl sie eigentlich aus ihrer Sicht unvernünftig ist. Dies auszuschließen würde bedeuten, dass man von der festen Gewissheit ausgeht, dass sich Experten partout nicht irren können und immer die Vernunft und mit ihr die beste Lösung obsiegt.

Realistischer ist die Vorstellung, dass sich auch EU-Gremien irren können. Niemand ist unfehlbar - gemäß dieser Erkenntnis hat die Demokratie übrigens in grauer Vorzeit den aufgeklärten Absolutismus abgelöst. Die Qualität einer legitimen politischen Ordnung bemisst sich schließlich auch an ihrer Fähigkeit, Fehler revidieren zu können. Wenn sich eine dem Wähler verantwortliche Regierung irrt, so kann der Souverän sie dafür bestrafen, indem er sie abwählt. Wenn sich Experten in EU-Gremien irren, könnte dies für die Irrenden ohne Folgen bleiben, weil man im Zweifel gar nicht weiß, wer genau den Fehler begangen hat $-\mathrm{zu}$ sehr hat man sich bei den einschlägigen Beratungen und in der Konsenssuche ,verstrickt“, um ex post irgendjemanden zur Verantwortung ziehen zu können. Wen soll man denn bestrafen, wen soll man an ihre Stelle setzen, wenn man mit der Performanz einer Deliberationsrunde nicht zufrieden war, und wer soll darüber auf Basis welcher Kriterien entscheiden? Schnell stößt man also auch auf das Problem, dass man nicht angeben kann, wer nach welchen Maßstäben objektiv beurteilen kann, ob ein Prozess so „deliberativ“ ist, dass man annehmen darf, er begünstige rationale Ergebnisse. Selbst in Höchst- bzw. Verfassungsgerichten oder aber in Geschworenen-Jurys, bei denen häufig unterstellt wird, dass hier ein besonders guter Nährboden für Deliberation existiert (exemplarisch: Rawls 1999: 139), ist der dominierende Interaktionsmodus zuweilen eher das „Verhandeln“ als das „Beraten“. Auch die Einbeziehung aller potenziell „Betroffenen“ in eine Beratung ist keine Garantie für deren deliberative Qualität. ${ }^{8}$ Möglicherweise können von einer Entscheidung auch Interessen betroffen sein, die man zuvor gar nicht wahrgenommen hat und die deswegen im Prozess der Deliberation gar nicht zu Gehör gekommen sind.

8 Möglichst viele bzw. sogar alle „Betroffene“ zu Gehör kommen zu lassen - ein wohlfeiles Standardargument der Deliberationstheoretiker - kann im Übrigen auch ungemein kontraproduktiv sein. Wie das häufig angeführte Beispiel der amerikanischen "Jurys" belegt, ist es dort für eine „unvoreingenommene“ Deliberation zwingend erforderlich, dass die Teilnehmer gerade nicht „,betroffen“ sind und sich auch von jenen, die tatsächlich „,betroffen“ sind, in keiner Weise beeinflussen lassen. 
Schließlich gilt ganz grundsätzlich, dass alle Modelle deliberativer Demokratie die Unterscheidung zwischen „rational“ und „,irrational“ machen müssen, da man nur entlang dieser Unterscheidung Selektionskriterien für eine vernünftige politische Willensbildung gewinnen kann (Young 2001b). Es gibt jedoch nicht nur objektiv „gute“ bzw. ,rationale“ und „schlechte“ bzw. „,irrationale“ Entscheidungen. Die Demokratie wurde auch deshalb erfunden, weil sich keiner anmaßen kann, zwischen objektiv guten oder schlechten Entscheidungen allgemein verbindlich unterscheiden und auf dieser Basis dann ,rational“ auswählen zu können. Es gibt schlechterdings keine Instanz, die im Besitz einer unumstößlichen Wahrheit ist.

Daraus resultiert ein weiteres Kardinalproblem: Selbst wenn eine kollektiv verbindliche Entscheidung nach allen Regeln der deliberativen Kunst getroffen wurde, kann es gleichwohl passieren, dass sie aus Sicht der Mehrheit der Bevölkerung trotz ihrer Vernünftigkeit keine Anerkennung findet und entsprechend abgelehnt wird (Fishkin 1997: 1 ff.). Möglicherweise ist die Bevölkerung nicht in der Lage und auch nicht willens, die „Wahrheit zu schauen“ und das Vernünftige immer und überall zu erkennen. In der Demokratie werden solche Wahrheitsfragen daher ganz bewusst ausgeklammert; was gut oder schlecht ist, entscheidet der Wähler, und dieser regierte Wähler irrt sich leider genauso oft wie der regierende Gewählte - aber immerhin hat der Wähler die Wahl, sich auch mal falsch entscheiden und seinen Irrtum beim nächsten Mal wieder korrigieren zu können. Der Wähler wird aber in einer deliberativen Demokratie offensichtlich immer weniger gebraucht.

\section{Fazit}

Die Paradoxie der Theorie der deliberativen Demokratie liegt im europäischen Verwendungszusammenhang auf der Hand. Sie ist überangepasst, weil sie die partiell zu beobachtende Entkopplung der supranationalen Entscheidungsträger nicht mehr als demokratisches Übel, sondern als deliberativen Mehrwert ansieht, den man schlechterdings nicht kritisieren kann. Sie ist realitätsentrückt, weil sie andererseits nicht erkennen will, dass die Interaktionsmodi in europäischen Entscheidungsprozessen nur in Ausnahmefällen den Grundsätzen deliberativer Demokratie entsprechen. Insofern ist diese Theorie, die in der Debatte um legitimes Regierens jenseits des Nationalstaats eine hegemoniale Stellung beansprucht, eigentümlich wirklichkeitsfern, also unwirklich, und daher auch nicht vernünftig. 
In „Wirklichkeit“ zeigt sich, dass überall dort, wo die sogenannten „vested interests" auf dem Spiel stehen, wo es um Verteilungsfragen, Werte und schließlich um Machtfragen geht, der dominierende Interaktions- und Entscheidungsmodus interessenorientiertes „bargaining“ in hochgradig rückgekoppelten intergouvernementalen Verhandlungssystemen ist, in denen jeder Verhandlungsteilnehmer sein Veto einlegen kann. Mag man mit den dort getroffenen Entscheidungen aus Output-Perspektive nicht immer zufrieden sein, so kann man sich wenigstens aus Input-Perspektive damit trösten, dass die Präferenzen von Wählerinnen und Wählern in der Regel nicht einfach ignoriert werden können.

Exakt dies ist aber generell das Signum von Demokratien - unabhängig davon, ob man es mit mehrheits- oder konsensdemokratischen Demokratie zu tun hat: Die beste Lösung für alle zu finden ist schwierig, daher müssen wir damit leben, dass es oft nur zweit- und drittbeste Lösungen gibt, manchmal auch gar keine. Solange aber die zugegeben manchmal etwas naive Hoffnung besteht, dass es die „Anderen“ beim nächsten Mal besser machen als die jetzt Regierenden, können wir mit der Demokratie ganz gut leben. Wir müssen aber weiterhin zumindest die Wahl zwischen Alternativen haben. Wie diese auf EU-Ebene organisiert werden könnte, wäre gerade vor dem Hintergrund der erschütternd niedrigen Wahlbeteiligung bei den EP-Wahlen $2009^{9}$ allemal ein lohnenswerteres Thema für eine europäische Demokratietheorie als ein Glaubensbekenntnis zugunsten einer deliberativen Demokratie, die in der real existierenden Welt des Politischen allenfalls in Spurenelementen anzutreffen ist. Eines scheint vor diesem Hintergrund sicher: Um das drängende Problem des legitimen und demokratischen Regierens jenseits des Nationalstaats befriedigend lösen zu können, wird sich die Politikwissenschaft weiter anstrengen müssen.

\section{Literatur}

Benhabib, Seyla, 1996: Toward a Deliberative Model of Democratic Legitimacy, in: dies. (Hrsg.), Democracy and Difference. Contesting the Boundaries of the Political, Princeton, 67-94.

Böckenförde, Ernst-Wolfgang, 1987: Demokratie als Verfassungsprinzip, in: Josef Isensee/Paul Kirchhof (Hrsg.), Handbuch des Staatsrechts, Band I, Heidelberg, 887-952.

9 Die Wahlbeteiligung ist 2009 europaweit auf ein Rekordtief von $43 \%$ gesunken. Vgl. http://www.dw-world.de/dw/article/0,,4310053,00.html. 
Bohman, James, 1997: Deliberative Democracy. Essays on Reason and Politics, Cambridge.

Bohman, James, 2005: From demos to demoi: Democracy across borders, in: Ratio Juris 18, 293-314.

Brunkhorst, Hauke, 2003: Der lange Schatten des Staatswillenspositivismus. Parlamentarismus zwischen Untertannenrepräsentation und Volkssouveränität, in: Leviathan 31, 362-381.

Buchstein, Hubertus/Jörke, Dirk, 2003: Das Unbehagen an der Demokratietheorie, in: Leviathan 31, 470-495.

Chambers, Simone, 2003: Deliberative democratic theory, in: Annual Review of Political Science 6, 302-326.

Cohen, Joshua, 1989: Deliberation and democratic legitimacy, in: Alan Hamlin/ Philip Pettit (Hrsg.), The Good Polity, Oxford, 17-34.

Dryzek, John S., 2006: Deliberative Global Politics. Discourse and Democracy in a Divided World, Cambridge.

Dryzek, John S., 2007: Networks and Democratic Ideals: Equality, Freedom, and Communication, in: Eva Sorensen/Jacob Torfing (Hrsg.), Theories of Democratic Governance, Basingstoke, 262-273.

Elster, John, 1998: Deliberative Democracy, Cambridge.

Eriksen, O., 2005: Making the European Polity: Reflexive Integration in the EU, London.

Fishkin, James S., 1997: The Voice of the People. Public Opinion and Democracy, New Haven.

Follesdal, Andreas/Hix, Simon, 2005: Why There is a Democratic Deficit in the EU: A Response to Majone and Moravcsik, European Governance Papers, No. C-05-02, EUROGOV.

Fossum, John E./Trenz, Hans-Jörg, 2006: The EU's Fledgling Society: From Deafening Silence to Critical Voice in European Constitution-Making, in: Journal of Civil Society 2, 57-77.

Frisch, Annika, 2007: Das Potenzial deliberativer Demokratietheorie für die Konzeptionalisierung von Demokratie in der Europäischen Union, in: ZPol 17, 711-733.

Gerstenberg, Oliver/Sabel, Charles, 2001: Direct Deliberative Polyarchy: An Institutional Ideal for Europe?, in: Renaud Dehousse/Christian Joerges (Hrsg.), Good Governance and Administration in Europe's Integrated Market, Oxford, 289-342.

Habermas, Jürgen, 1962: Strukturwandel der Öffentlichkeit, Neuwied.

Habermas, Jürgen, 1973: Legitimationsprobleme im Spätkapitalismus, Frankfurt a. M. 
Habermas, Jürgen, 1998: Die postnationale Konstellation, Frankfurt a. M.

Habermas, Jürgen, 1992: Faktizität und Geltung. Beiträge zur Diskurstheorie des Rechts und des demokratischen Rechtsstaats, Frankfurt a. M.

Hegel, Georg Wilhelm Friedrich, 1972: Grundlinien der Philosophie des Rechts, Frankfurt a. M.

Heller, Hermann, 1992: Gesammelte Schriften, in Verbindung mit Martin Drath herausgegeben von Christoph Müller, Band 1, Tübingen (2. durchgesehene und um ein Nachwort erweiterte Aufl.; insges. 3 Bände).

Hitzel-Cassagnes, Tanja, 2004: Geltung und Funktion. Supranationale Gerichtsbarkeit im Spannungsfeld von praktischer Rationalität, Recht und Demokratie, Baden-Baden.

Höreth, Marcus, 1999: Die Europäische Union im Legitimationstrilemma, Baden-Baden.

Joerges, Christian/Neyer, Jürgen, 1997: From International Bargaining to Deliberative Political Processes: The Constitutionalisation of Comitology, in: European Law Journal 3, 272-299.

Joerges, Christian/Neyer, Jürgen, 1998: Vom intergouvernementalen Verhandeln zur deliberativen Politik, in: Beate Kohler-Koch (Hrsg.), Regieren in entgrenzten Räumen, PVS-Sonderheft 29, 207-234.

Jörke, Dirk, 2003: Demokratie als Erfahrung. John Dewey und die politische Philosophie der Gegenwart, Wiesbaden.

Moravcsik, Andrew, 2004: Is there a democratic deficit in world politics?, in: Government and Opposition 39 (2), 336-363.

Müller, Harald, 1994: Internationale Verhandlungen als kommunikatives Handeln. Zur Kritik der utilitaristischen Handlungstheorien, in: Zeitschrift für Internationale Beziehungen 1, 15-44.

Neyer, Jürgen, 2006: The Deliberative Turn in Integration Theory, in: Journal of European Public Policy 13, 779-791.

Neyer, Jürgen, 2007: Welche Integrationstheorie braucht Europa?, in: integration 4, 382-393.

Niesen, Peter, 2007: Deliberation ohne Demokratie? Zur Konstruktion von Legitimität jenseits des Nationalstaats (unveröffentlichtes Manuskript).

Nullmeier, Frank, 2008: Die Delegitimation von Wahlen, Paper präsentiert auf der Drei-Länder-Tagung, 21.-23.11.2008, Universität Osnabrück.

Offe, Claus, 2005: Rote Fäden und lose Enden. Bemerkungen zu einer MegaAgenda, in: Anna Geis/DavidStrecker (Hrsg.), Blockaden staatlicher Politik, Frankfurt a. M./New York, 245-277. 
Parkinson, John, 2003: Legitimacy Problems in Deliberative Democracy, in: Political Studies 51, 180-196.

Rawls, John, 1999: A Theory of Justice, Cambridge.

Risse, Thomas, 2004: Global Governance and Communicative Action, in: Government and Opposition 39 (2), 288-313.

Scharpf, Fritz, 1970: Demokratietheorie zwischen Utopie und Anpassung, Konstanz.

Schmalz-Bruns, Rainer, 1999: Deliberativer Supranationalismus. Demokratisches Regieren jenseits des Nationalstaats, in: Zeitschrift für Internationale Beziehungen 6 (2), 185-244.

Schmalz-Bruns, Rainer, 2002: Deliberative Politik als Modell demokratischen Regierens jenseits des Nationalstaates, in: Matthias Lutz-Bachmann/James Bohman (Hrsg.), Weltstaat oder Staatenwelt?, Frankfurt a. M., 248-273.

Shapiro, Ian, 1999a: Democratic Justice, New Haven.

Shapiro, Ian, 1999b: Enough of Deliberation. Politics is about Interests and Power, in: Stephen Macedo (Hrsg.), Deliberative Politics: Essays on ,Democracy and Disagreement', New York, 28-38.

Shapiro, Ian, 2002: Optimal Deliberation?, in: The Journal of Political Philosophy 10, 196-211.

Sirianni, Carmen, 2001: Civic Innovation in America, Berkeley.

Sonnicksen, Jared, o. J.: Deliberative Democracy as a Model for the EU. Normative implications of adapting democracy to governance beyound the nation-state (unveröffentlichtes Manuskript).

Sørensen, Eva/Torfing, Jacob, 2005: The Democratic Anchorage of Governance Networks, in: Scandinavian Political Studies 28, 195-218.

Young, Iris M., 2001a: Activist Challenges to Deliberative Democracy, in: Political Theory 5, 670-690.

Young, Iris M., 2001b: Inclusion and Democracy, Oxford. 
Korrespondenzanschrift:

PD Dr. Marcus Höreth

Seminar für Politikwissenschaft

Universität Göttingen

Platz der Göttinger Sieben 3

37073 Göttingen

E-Mail: marcus.hoereth@sowi.uni-goettingen.de

\section{Wahlforschung aktuell}

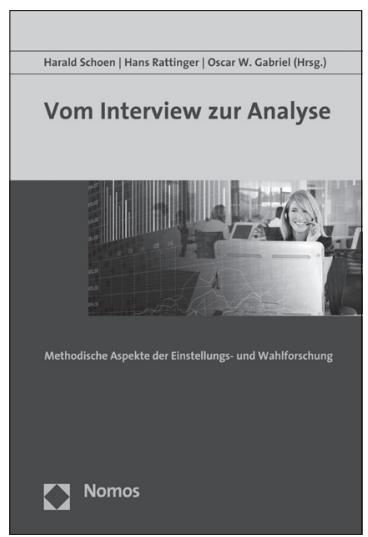

\section{Vom Interview zur Analyse}

Methodische Aspekte der Einstellungs- und

Wahlforschung

Herausgegeben von Harald Schoen, Hans Rattinger und Oscar W. Gabriel 2009, 390 S., brosch., 44,-€, ISBN 978-3-8329-4725-5

Der vorliegende Band widmet sich ausgewählten Fragen zur Methodik der Wahl- und Einstellungsforschung.

In 15 Kapiteln werden auf der Grundlage umfangreichen Datenmaterials, das seit der Wiedervereinigung in Ost- und Westdeutschland gesammelt wurde, zentrale Themen der Forschung behandelt. 